\title{
Évolution des coopératives laitières en Pologne
}

Point de comparaison avec quelques aspects de la législation française

\section{Evolution of dairy cooperatives in Poland} A point of comparison with some aspects of French legislation

\section{Danuta Mierzwa}

Numéro 298, novembre 2005

URI : https://id.erudit.org/iderudit/1021841ar

DOI : https://doi.org/10.7202/1021841ar

Aller au sommaire du numéro

\section{Éditeur(s)}

Institut de l'économie sociale (IES)

\section{ISSN}

1626-1682 (imprimé)

2261-2599 (numérique)

Découvrir la revue

\section{Citer cet article}

Mierzwa, D. (2005). Évolution des coopératives laitières en Pologne : point de comparaison avec quelques aspects de la législation française. Revue internationale de l'économie sociale, (298), 56-74.

https://doi.org/10.7202/1021841ar
Résumé de l'article

L'efficacité économique d'une coopérative laitière résulte de sa position sur le marché des produits laitiers et de son rôle dans l'industrie agroalimentaire. Cet article présente le contexte de l'agriculture en Pologne, avant de s'intéresser plus spécifiquement aux coopératives. Parmi les obstacles à leur développement, le texte souligne une capacité d'autofinancement limitée. L'afflux de capitaux extérieurs et la mise en place de structures plus flexibles, notamment par le biais d'alliances, sont indispensables. Pour conserver son potentiel sur le marché du lait et faire face à la compétition européenne et mondiale, la Pologne devra aussi utiliser tous les moyens financiers accordés dans le cadre de l'intégration à l'Union européenne. Selon l'auteur, l'expérience de la France pourrait devenir une sorte de modèle pour la restructuration et la modernisation du secteur coopératif en Pologne. Une réforme des règles juridiques polonaises a d'ailleurs été proposée, en s’inspirant de cette expérience française. 


\section{ÉVOLUTION DES COOPÉRATIVES LAITIËRES EN POLOGNE}

Point de comparaison avec quelques aspects de la législation française

$\left({ }^{*}\right)$ Chaire d'économie et d'organisation de l'agriculture, Académie agricole de Wroclaw. E-mail: mierzwa@ekonom.ar.wroc.pl.

\author{
par Danuta Mierzwa ${ }^{(*)}$
}

L'efficacité économique d'une coopérative laitière résulte de sa position sur le marché des produits laitiers et de son rôle dans l'industrie agroalimentaire. Cet article présente le contexte de l'agriculture en Pologne, avant de s'intéresser plus spécifiquement aux coopératives. Parmi les obstacles à leur développement, le texte souligne une capacité d'autofinancement limitée. L'afflux de capitaux extérieurs et la mise en place de structures plus flexibles, notamment par le biais d'alliances, sont indispensables. Pour conserver son potentiel sur le marché du lait et faire face à la compétition européenne et mondiale, la Pologne devra aussi utiliser tous les moyens financiers accordés dans le cadre de l'intégration à l'Union européenne. Selon l'auteur, l'expérience de la France pourrait devenir une sorte de modèle pour la restructuration et la modernisation du secteur coopératif en Pologne. Une réforme des règles juridiques polonaises a d'ailleurs été proposée, en s'inspirant de cette expérience française.

'intégration de la Pologne à l'Union européenne a constitué un objectif majeur de la politique polonaise. Elle concerne tous les Polonais, qu'ils vivent en Pologne ou en dehors du pays. La constitution d'une union des pays d'Europe se matérialise par la mise en œuvre d'actions concrètes répondant à de réelles attentes. Mais il faut également faire face aux obstacles nés de la confrontation entre les pays européens actuels et les nouveaux pays économiquement retardés, voire pauvres, du centre et de l'est de l'Europe.

Depuis quelques années déjà, les coopératives polonaises subissent les conséquences de la transformation de leur économie, mais surtout se trouvent de plus en plus confrontées à la concurrence de l'agriculture moderne de l'UE et de ses organisations agricoles qui tendent à conquérir de nouveaux marchés. Dans cette perspective, il faut répondre à plusieurs problèmes d'ordre économique, financier et organisationnel. A ce niveau, nous manquons de modèles à appliquer pour réaliser ces transformations. En revanche, l'expérience des pays de l'UE peut être un atout pour l'adaptation de certaines activités des coopératives polonaises et un point de départ à l'établissement d'un modèle général de coopérative. 
(1) Danuta Mierzwa a fait des stages scientifiques dans plusieurs structures coopératives en France; elle a consulté les spécialistes, les responsables des coopératives; elle a visité plusieurs fermes situées dans différentes régions françaises; elle a identifié les problèmes de ces agriculteurs-membres de la coopérative. Actuellement, elle travaille avec René Mauget, de l'Essec-Imia, à Paris. Elle a donc pu prendre connaissance des modèles français et de leur intérêt pour la résolution des problèmes polonais.
La littérature économique manque d'informations précises sur ce thème. Pour compléter au moins en partie ce manque d'information, l'article s'intéresse aux réalisations françaises ${ }^{(1)}$.

La France a une forte tradition agricole. La coopération agricole et les organisations coopératives n'y sont pas étrangères. Les coopératives agricoles occupent en effet une position essentielle dans l'industrie agroalimentaire et sont présentes sur tout le territoire français. La structure organisationnelle du secteur coopératif est très riche et diversifiée. Cette structure a été créée par les agriculteurs, dans leur propre intérêt. Les coopératives forment une structure verticale par région, tandis que les unions nationales rassemblent ces coopératives au niveau national. En ce qui concerne leur activité, les coopératives peuvent intégrer d'autres unités de production de la même branche pour former des coopératives nationales ou européennes. Dans le domaine financier, elles peuvent s'unir avec des coopératives d'autres branches et même avec des entreprises privées.

Cependant, les expériences françaises ne peuvent pas constituer les seuls modèles de la restructuration des coopératives polonaises.

\section{Présentation de l'agriculture polonaise}

Les coopératives jouent en Pologne un rôle important dans le secteur laitier. Cependant, leurs divisions entraînent un manque d'efficacité et empêchent une intégration de la production laitière. L'état actuel de l'agriculture polonaise est également un facteur défavorable qui ne participe pas à l'amélioration de l'industrie laitière. L'activité d'élevage se trouve principalement au sud-ouest et au centre de la Pologne. La part de l'agriculture dans le PNB a baissé d'année en année pour atteindre 2,9\% en 2001 (tableau 1).

Tableau 1
Part de l'agriculture, élevage et sylviculture dans l'économie nationale
\begin{tabular}{l|cccccccc} 
PNB & $\mathbf{1 9 9 5}$ & $\mathbf{1 9 9 6}$ & $\mathbf{1 9 9 7}$ & $\mathbf{1 9 9 8}$ & $\mathbf{1 9 9 9}$ & $\mathbf{2 0 0 0}$ & $\mathbf{2 0 0 1}$ \\
\hline$\%$ & 6,0 & 5,5 & 4,8 & 4,1 & 3,4 & 3,3 & 2,9
\end{tabular}

Source: Agriculture et économie alimentaire en Pologne, ministère de l'Agriculture et du Développement de la campagne, 2001.

En Pologne, les surfaces cultivées représentent 18,4 millions d'hectares, ce qui constitue presque $59 \%$ de la surface du pays. Les sols ne sont pas parmi les plus fertiles d'Europe. Un peu plus de $3 \%$ des terres arables appartiennent aux classes I et II, et presque $35 \%$ aux classes V et VI. La surface totale des terres arables comprend les prairies, qui couvrent 12,9\% de cette surface. Si certaines régions sont prédisposées à l'élevage du bétail laitier, la structure du troupeau ne correspond pas aux potentialités de 
l'économie. La grande dispersion des exploitations agricoles est également un facteur supplémentaire qui vient expliquer l'état actuel de l'agriculture polonaise, et plus particulièrement celui de l'industrie laitière. La surface moyenne d'une exploitation individuelle en 2001 était de 9 hectares, par rapport à 19,4 hectares en moyenne dans l'Union européenne. La structure des exploitations agricoles individuelles, en pourcentage de la surface totale, se présentait comme suit (tableau 2).

\section{Tableau 2}

Structure des exploitations en 2001

\begin{tabular}{l|ccccc}
$\begin{array}{l}\text { Exploitations agricoles } \\
\text { par types de surface (en \%) }\end{array}$ & $\mathbf{1 - 2}$ ha & $\mathbf{2 - 5}$ ha & $\mathbf{5 - 1 0}$ ha & $\mathbf{1 0 - 1 5}$ ha & $>\mathbf{1 5}$ ha \\
\hline & 22,8 & 33,8 & 24,3 & 9,7 & 9,4
\end{tabular}

Source: Agriculture et économie alimentaire en Pologne, ministère de l'Agriculture et du Développement de la campagne, 2001.

(2) Les chiffres entre crochets renvoient à la bibliographie publiée en fin d'article.
La production animale en Pologne repose principalement sur l'élevage des bovins et des porcins. On constate que la capacité laitière s'est détériorée $\left[15^{(2)}\right.$. De plus, plusieurs troupeaux de vaches ont été éliminés après la transformation du système politique. La réduction du cheptel bovin depuis 1974 a entrainé une baisse du nombre de vaches d'environ 45 \% sur les dernières années (tableau 3). Cette baisse se poursuit [13]. Les districts où l'on élève le plus de bétail bovin sont ceux de Podlachie, de Petite Pologne, de Grande Pologne, de Swietokrzyskie et de Mazovie. Ce sont les laiteries situées sur ces terres qui fournissent le plus de matières à l'industrie laitière.

\section{Tableau 3}

Evolution du cheptel bovin entre 1996 et 2001 (en milliers)

\begin{tabular}{l|c|cc}
\multirow{2}{*}{ Année } & Ensemble du cheptel & \multicolumn{2}{|c}{ Répartition } \\
\hline 1996 & 3442 & 3361 & Secteur public \\
1997 & 3496 & 3418 & 81 \\
1998 & 3471 & 3400 & 78 \\
1999 & 3299 & 3237 & 71 \\
2000 & 3047 & 2996 & 62 \\
2001 & 2991 & 2945 & 51 \\
& & & 46
\end{tabular}

Source: marché laitier, 2000. 
La réduction du cheptel bovin risque de se poursuivre, mais à un rythme moins élevé. Elle sera accompagnée de la concentration de l'élevage dans de grandes exploitations agricoles. Ce phénomène est favorisé par la demande, de la part de l'industrie laitière, d'une meilleure qualité pour le lait et par la course à la rentabilité de la production laitière [17].

En Pologne, la production laitière est couverte par 1,2 million d'exploitations et la production commerciale par 400000 exploitations fournissant des matières à l'industrie. La structure du cheptel bovin en Pologne n'est pas favorable à son propre développement. Ainsi, $60 \%$ des fournisseurs sont propriétaires d'une ou deux vaches, $25 \%$ en ont entre trois et cinq et $15 \%$ en possèdent de six à dix. Seulement un faible pourcentage d'agriculteurs sont propriétaires de plus de dix vaches. Pour améliorer la production du secteur laitier, il est donc nécessaire de le restructurer par le regroupement des exploitations agricoles.

La production et l'achat de lait, dynamiques dans années 70 et 80 , ont commencé à se détériorer en 1990 (tableau 4).

\section{Tableau 4 \\ Production et achat de lait (en millions de litres)}

\begin{tabular}{lccc} 
Année & Production & $\begin{array}{c}\text { Vente totale, production } \\
\text { de marchandises }\end{array}$ & $\begin{array}{c}\text { Achat pour } \\
\text { le secteur laitier }\end{array}$ \\
\hline 1989 & 15926 & 11723 & 11385 \\
1992 & 12770 & 8848 & 6696 \\
1996 & 11355 & 7955 & 6315 \\
1998 & 12178 & 8788 & 7006 \\
2000 & 11494 & 8101 & 6487 \\
2001 & 11538 & 8448 & 6832 \\
\hline
\end{tabular}

La qualité du lait acheté s'améliore régulièrement. Selon les études de l'Inspection des achats et de la transformation des produits agricoles, la part du lait de la classe extra dans l'achat total de lait a augmenté en 2001 pour atteindre $67 \%$, alors qu'elle ne s'élevait qu'à $56 \%$ en 2000 . On observe donc une augmentation régulière de la part de la classe extra jusqu'en 2001, et les prévisions sont favorables.

Les producteurs de lait polonais ont la possibilité d'atteindre la classe extra grâce aux crédits préférentiels de l'Agence de restructuration et de modernisation de l'agriculture et aux fonds d'assistance créés par plusieurs coopératives pour l'équipement technique des exploitations spécialisées dans la production de lait. A l'avenir, les laiteries pourront ainsi acheter essentiellement du lait de la classe extra pour la transformation [1].

Entre 1990 et 2000, on a observé une augmentation de la production de lait par vache, dans les exploitations privées, mais aussi dans le secteur public (tableau 5). La production moyenne a commencé à augmenter à partir 
de 1993. Elle est pourtant beaucoup plus basse que celle des pays européens qui ont la production laitière la plus élevée. Si cette production par vache poursuit son augmentation dans les grandes exploitations, elle est plus mitigée dans les autres types d'exploitation [17]. Dans les années à venir, la production laitière en Pologne devrait augmenter. Ce phénomène devrait être amplifié par la restructuration et la modernisation de l'amont, stimulées par des crédits préférentiels.

\section{Tableau 5}

Production par vache

\begin{tabular}{l|ccc}
\multirow{2}{*}{ Année } & \multicolumn{3}{|c}{ Capacité laitière (en litres par vache) } \\
& Valeur nationale moyenne & Entreprises individuelles & Secteur public \\
\hline 1989 & 3260 & 3156 & 4021 \\
1990 & 3151 & 3042 & 4035 \\
1992 & 3015 & 2942 & 3704 \\
1994 & 3121 & 3072 & 3822 \\
1996 & 3249 & 3210 & 4227 \\
1998 & 3491 & 3443 & 5000 \\
2000 & 3668 & 3605 & 5175 \\
2001 & 3828 & 3758 & 5742
\end{tabular}

Le lait et les produits laitiers sont essentiels dans la mesure où ils ont une valeur alimentaire, diététique et économique incontournable. Le lait et les produits laitiers ont toujours joué un rôle déterminant dans le modèle alimentaire polonais. Les années 90 ont montré une consommation annuelle qui dépassait les 200 litres par habitant. Mais cette quantité n'a cessé de baisser depuis 2000, pour aboutir à une consommation de 185 litres par habitant en 2001 (tableau 6). Cette baisse a été causée par la détérioration du revenu de la plupart de groupes socio-économiques, par la diminution de l'offre et par l'augmentation des prix de détail de plusieurs produits laitiers. A cela s'ajoutent une augmentation de l'export et une diminution de l'import.

\section{Les coopératives en Pologne}

En Pologne, la branche laitière joue un rôle stratégique dans l'alimentation de la population et occupe une position majeure dans l'économie alimentaire. Les coopératives ont commencé à se développer durant la seconde moitié du XIX siècle. Elles ont ensuite pris leur essor dans la période 
Tableau 6

Consommation de lait par habitant

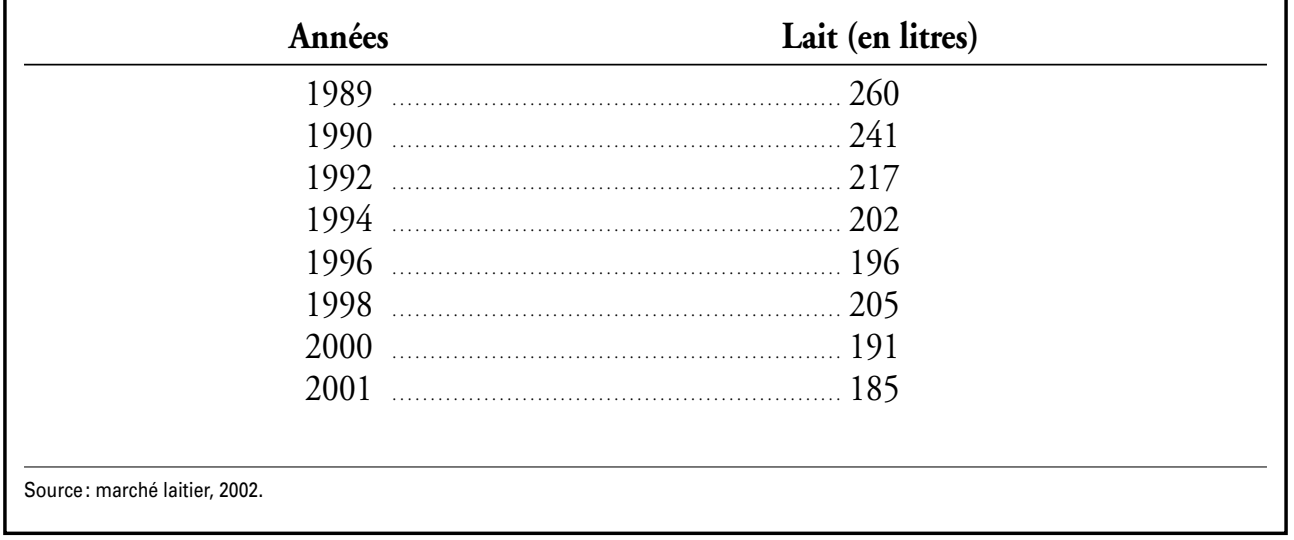

de l'entre-deux-guerres et pendant les premières années qui ont suivi la Seconde Guerre mondiale. Les coopératives ont été supprimées en 1950, pour renaitre en 1957.

Jusqu’à la fin de 1989, la coopérative polonaise a constitué une structure à trois niveaux. Son unité fondamentale était composée de coopératives laitières d'arrondissement dont les membres étaient des agriculteurs. Le deuxième niveau était constitué d'associations de district auxquelles appartenaient les coopératives d'arrondissement. Le troisième niveau, enfin, était formé par l'Union centrale des coopératives laitières, où se regroupaient des coopératives de deuxième niveau et celles qui n'appartenaient pas aux structures coopératives régionales ou d'arrondissement. Jusqu'en 1990, les coopératives avaient une exclusivité pour l'achat et la transformation alimentaire du lait. La mutation du système politique, c'est-à-dire la transition de l'économie planifiée à l'économie libérale après 1989, puis la réforme des organisations commencée par la loi sur les changements d'organisation et d'activité coopérative du 20 janvier 1990 ont entrainé des modifications dans le fonctionnement de la société. On a supprimé des coopératives centrales et des associations coopératives régionales, alors que des coopératives de premier niveau ont obtenu une autonomie plus grande et sont devenues indépendantes des structures suprafondamentales coopératives, administratives et politiques. La loi en vigueur a introduit une nouvelle situation: les coopératives ont été privées de sources d'approvisionnement et ont rompu leurs liens économiques, coopératifs et commerciaux avec d'autres organisations coopératives et nationales. Elles ont également perdu l'aide juridique professionnelle dont elles disposaient et le conseil d'associations de patronage. Elles ont aussi été privées de subventions et d'assistances financières. Les coopératives ont dû prendre des décisions importantes pour leur transformation, alors que le contexte économique et leur situation 
organisationnelle et financière étaient instables. A cela est venue s'ajouter la situation politique du pays. On considérait que le secteur coopératif était une création du régime communiste, qui devait donc être désormais privatisée. En effet, avant 1990, les coopératives avaient obtenu de l'Etat d'importantes subventions pour la production. Par la suite, les coopératives ont été forcées de rechercher par elles-mêmes des moyens financiers [9]. Le nombre de coopératives entre 1989 et 2001 a ainsi évolué (tableau 7).

\section{Tableau 7}

Evolution du nombre de coopératives entre 1989 et 2001

\begin{tabular}{lccccc} 
& $\mathbf{1 9 8 9}$ & $\mathbf{1 9 9 0}$ & $\mathbf{1 9 9 4}$ & $\mathbf{1 9 9 8}$ & $\mathbf{2 0 0 1}$ \\
\hline Nombre de coopératives laitières & 340 & 348 & 303 & 285 & 220 \\
Nombre d'entreprises laitières & 704 & 714 & 334 & 350 & 380
\end{tabular}

Source: marché laitier, 2002.

Après 1990, on note une tendance à la baisse du nombre de coopératives. Cette baisse est due à la disparition de certaines unités et devrait se poursuivre parallèlement au processus d'intégration de la Pologne à l'Union européenne. En 2001, on dénombrait en Pologne environ 400 entreprises laitières, dont 280 employaient plus de cinquante personnes, soit un total de 48600 personnes, ce qui représente cependant une baisse de 8,2\% par rapport à l'année précédente. La situation économique des coopératives s'est détériorée. La rentabilité brute en 2001 est en baisse par rapport à 2000 [17].

La suppression des structures supracoopératives a conduit à la désintégration du secteur coopératif et à l'affaiblissement des unités coopératives fondamentales. Les coopératives laitières d'arrondissement se sont vite rendu compte que, séparément, elles n'étaient pas capables de faire face à la dégradation de l'ensemble du secteur laitier. Le 11 novembre 1991 a été créée l'Association nationale des coopératives laitières, à laquelle ont adhéré des coopératives de toute la Pologne. A cela s'ajoute la mise en place de syndicats régionaux de coopératives pour résoudre efficacement les problèmes de l'industrie laitière polonaise [9].

Il existe également une organisation qui associe les entreprises privées de transformation laitière: l'Union des entreprises de transformation laitière. Créée en juin 1999, elle a été fondée par les entreprises suivantes: Lindals, Nestlé Polska, Danone, Hochland Polska, Toska, Elmilk, Osmos, Ovita Nutricia, Mitex et Obi. Actuellement, elle compte dix-huit entreprises privées de transformation laitière et certaines entités qui achètent du lait au secteur laitier polonais. A part les membres fondateurs, l'union se compose d'autres sociétés à responsabilité limitée: Arla Foods, BEL Polska, Lactalis, Sertop Tyhy, Agrocomex, Fromako, Warmia Dairy, Mleko 
Pomorskie, Lactima, Campina Polska, ZOTT Polska et Westland-Sery. Les entreprises regroupées dans cette union accordent une très haute importance à la qualité de leurs produits. La plupart des entreprises possèdent le système HACCP; certaines montrent même des systèmes de gestion dont la qualité est compatible avec les standards ISO 9000 et elles ont le certificat permettant d'exporter vers l'UE.

L'Union des entreprises privées de transformation laitière, par la coopération avec d'autres organisations laitières, a pour objet de créer un lobby représentant les intérêts des laiteries polonaises tant en Pologne qu'à l'étranger. En Pologne, elle coopère avec l'Association nationale des producteurs laitiers, l'Union nationale des coopératives laitières et l'Association des ingénieurs et techniciens de l'industrie alimentaire. Elle fait également partie de la Fédération internationale laitière et elle a commencé à coopérer avec l'Association européenne laitière.

L'un des nombreux obstacles au développement du secteur laitier polonais est la saisonnalité de la production laitière. Les coopératives ne bénéficient pas d'une capacité productive stable. Leurs coûts de production s'accroissent et, ainsi, leur compétitivité s'affaiblit sur les marchés nationaux et étrangers. Le système coopératif laitier joue un rôle important, bien que son état actuel requière un processus de modernisation important.

Actuellement, il est possible de dégager différents segments dans la transformation des produits laitiers:

- la production des produits laitiers traditionnels (lait alimentaire, crème, beurre), dominée par le système coopératif;

- la production de lait en poudre, de fromages de goût et de desserts laitiers, dominée par le système coopératif;

- la production de fromages fondus, segment légèrement dominé par des entreprises privées;

- la production de yaourts et de glaces, où dominent les entreprises privées [18]. La domination des entreprises privées et à capitaux étrangers dans le dernier segment représente une menace pour la transformation alimentaire nationale. Les entreprises à capitaux étrangers possèdent $30 \%$ du marché laitier polonais. On estime la part de Danone (capitaux français) à $30 \%$ sur le marché des yaourts et des desserts, à $45 \%$ sur le marché des fromages frais et à $22 \%$ sur le marché de la crème. L'entreprise hollandaise Campina a aussi une position forte sur le marché du yaourt et des desserts laitiers. L'entreprise allemande Hochland gagne de plus en plus de terrain. Parmi les autres entreprises investissant sur le marché polonais, on peut citer la danoise Arla Foods, les hollandaises Hoog wegt (fromage et lait en poudre) et Nemico (lait alimentaire), la suisse Molly (fromage et beurre) et l'américaine Land O'Lakes (fromage et beurre). Les entreprises s'étant retirées récemment sont la hollandaise Friesland et la française Yoplait. Ce phénomène menace la place des entreprises polonaises sur leur propre marché [3]. La situation financière difficile des entreprises polonaises ne leur permet pas de faire face à la compétition des entreprises étrangères. Une ligne technologique moderne leur est par exemple inaccessible. 
Le fait que la part des entreprises achetant de grandes quantités augmente est favorable et indique une concentration de l'industrie. Pourtant, le niveau d'avancement du processus de concentration de la production laitière n'est pas comparable à celui de l'UE. En effet, seules les entreprises produisant plus de 100 millions de litres de lait par an jouissent d'une forte position compétitive et d'un fort potentiel de développement. Or, parmi toutes les laiteries, dix seulement atteignent une telle production. C'est ainsi que la politique de l'Etat devient le facteur clef qui peut permettre la restructuration du secteur afin d'accélérer le processus de concentration. Par la création d'instruments efficaces, elle peut aider à construire des entreprises fortes et compétitives par rapport aux entreprises étrangères.

\section{Les problèmes des coopératives et les moyens de les résoudre}

L'intégration à l'UE, avec le statut de membre à part entière, constitue un objectif stratégique majeur de la politique économique et des affaires étrangères de la Pologne. L'agriculture est un secteur où les adaptations sont très nombreuses, mais nécessaires. Lindustrie laitière a une position importante dans le secteur agroalimentaire.

Le processus d'intégration au marché présente divers problèmes qui doivent être résolus de manière urgente. Voici les problèmes les plus importants à résoudre:

- Une mise à niveau par rapport aux standards de qualité du lait et de ses produits dérivés, ce qui veut dire l'observation des standards de qualité relatifs à la matière première, à son achat et à sa transformation, jusqu'aux produits finaux et à la vente aux clients. Deux axes sont à privilégier : l'accélération de la restructuration du secteur fournissant la matière de base, pour atteindre la qualité exigée par l'UE; la modernisation du processus d'achat de lait et l'organisation de réseaux de laboratoires pour arriver à un contrôle permanent de la qualité de la totalité du lait acheté.

- L'adaptation aux exigences de l'UE du système d'intervention sur le marché laitier, c'est-à-dire l'amplification du système actuel et l'adaptation des institutions d'intervention.

- L'élaboration d'un modèle et la construction d'un appareil efficace pour la gestion et le contrôle des quotas laitiers.

Ce sont des problèmes importants qui exigent de grands investissements de la part des producteurs et des acteurs de la transformation alimentaire. L'accélération de la modernisation du secteur laitier, indispensable pour l'intégration à l'UE, sera difficile sans un secours financier, car la rentabilité du secteur laitier est insuffisante pour engager de tels investissements [4].

Les standards qualité du secteur laitier polonais et le niveau d'exigence - peu élevé - pour le lait acheté sont les grands défis à relever. Ces critères atteignent un niveau très élevé dans I'UE. En Pologne, une nouvelle norme sur l'achat de lait cru: « Polska Norma » (dont le symbole est PN-A-86002: 1999), est entrée en vigueur le $1^{\text {er }}$ janvier 2000 . A cela s'ajoute la réforme 
du ministre des Finances sur les conditions d'acquisition et de transformation du lait, mise en place le 26 octobre 2002. Les agriculteurs qui veulent vendre du lait aux laiteries doivent se plier aux règles imposées. Ces laiteries achèteront du lait de la classe II jusqu'en 2002 et du lait de classe I jusqu'en 2006. Ce n'est que plus tard que le lait de la classe extra deviendra la norme [5]. En 2003, en Pologne, $67 \%$ du lait acheté appartient à la classe extra (la seule classe achetée dans l'UE).

Les standards qualité insuffisants des laiteries ont été la cause de l'arrêt forcé de l'export vers l'UE. Les inspecteurs de l'Union européenne ont divisé les exploitations polonaises en quatre catégories:

- A, exploitations répondant aux exigences de l'UE;

- B1, exploitations ayant de fortes chances de répondre à ces exigences;

- B2, exploitations qui auront besoin d'une période d'adaptation prolongée.

Elles peuvent solliciter une période de transition jusqu'en 2006;

- $\mathrm{C}$, exploitations ayant de faibles chances de répondre aux exigences de

l'UE. Elles ne pourront donc qu'acheter du lait.

Tableau 8

Exploitations polonaises en 2003 selon les catégories définies par l'UE

\begin{tabular}{l|cccc} 
Catégories définies par l'Union européenne & A & B1 & B2 & C \\
\hline Nombre d'entreprises & 36 & 156 & 108 & 90 \\
\hline Source: www.ppr.pl. & & & &
\end{tabular}

Un autre défi pour l'industrie laitière polonaise est la construction d'un appareil administratif efficace permettant de réguler le marché laitier. C'est un processus très coûteux, exigeant une préparation, des moyens financiers considérables pour l'équipement technique et l'emploi du personnel. En considérant le potentiel et l'importance du secteur laitier dans l'agriculture polonaise, le succès d'une telle opération pourrait avoir des retombées très positives pour toute l'économie nationale et pour les exploitations individuelles polonaises. Cependant, la période d'adaptation nécessite d'ores et déjà une préparation efficace et la mise en place de nombreuses activités pour résoudre les multiples problèmes qui surviennent. La mise en vigueur des régulations européennes exigera beaucoup d'investissements grâce auxquels il sera possible d'adapter les processus de production et de transformation du lait aux standards de l'UE.

L'un des instruments les plus importants de l'UE pour la régulation du marché laitier est le système des quotas. D'après les experts de l'UE, ce système assure l'équilibre du marché et fournit un soutien constant au secteur laitier. Le $1^{\text {er }}$ janvier 2002, une nouvelle loi sur la régulation du marché laitier et de ses produits est entrée en vigueur en Pologne. Cette loi est totalement adaptée aux régulations de l'UE. Elle concerne le système des quotas 
de la production laitière nationale, de l'achat et de la vente, le système d'intervention concernant certains produits laitiers, ainsi que les aides au stockage. La première phase d'application de ces quotas s'est étendue du $1^{\text {er }}$ avril 2004 au 31 mars 2005. Ainsi, les acteurs sont obligés d'envoyer aux présidents des services locaux des informations sur la quantité de lait acheté. La décision portant sur l'attribution des quotas laitiers individuels a été arrêtée le 31 janvier 2004.

Pour inverser cette proportion défavorable entre la production laitière et la consommation de lait, un Fonds de la promotion de l'industrie laitière a été créé en Pologne. Ses objectifs sont l'export, l'aide à l'organisation de salons et de foires, le soutien de projets scientifiques et l'amélioration de la qualité des produits laitiers.

La Commission européenne a proposé pour la Pologne un quota national fixé à 8875000 tonnes pour le lait, ce jusqu'en 2020. En considérant la consommation actuelle de lait en Pologne, c'est-à-dire la vente en gros (7900 000 tonnes), la vente directe (1 130000 tonnes) et le niveau courant d'autoapprovisionnement des exploitations agricoles (3200000 tonnes), ce quota permet d'utiliser le potentiel actuel de production de l'industrie laitière polonaise. Pourtant, il n'est pas tenable pour les années suivantes. On doit considérer que la demande de lait et de produits laitiers augmentera avec la hausse des revenus. Pour cette raison, la Pologne a proposé d'introduire un quota plus important et de l'augmenter à 11845000 tonnes en 2004 et 13740000 en 2008 [6].

L'un des problèmes des coopératives polonaises est le faible potentiel de l'industrie de transformation. La baisse la plus importante a été remarquée dans les laiteries du district de Basse Silésie. Pour prévenir ce phénomène, il faudrait se concentrer sur trois solutions: l'augmentation de la quantité de lait transformé, l'adaptation des capacités à la base de matières premières et le développement des ventes. Un autre problème du marché laitier est la faible importance du commerce de gros, ce qui entraîne une moindre qualité des produits et une difficulté pour transmettre des informations. Il manque aussi une plus grande coopération et une coordination des activités des coopératives, ce qui accentue la compétition entre elles. Le développement des supermarchés à capitaux étrangers constitue également un danger pour le réseau des coopératives. En effet, ce phénomène baisse progressivement le chiffre d'affaires des petits commerçants, y compris des coopératives. En prévention, les coopératives doivent créer des associations capables de satisfaire aux exigences des hyper et supermarchés où elles pourraient alors vendre leurs produits [19]. Le manque de moyens financiers est un obstacle majeur au développement. L'écart technologique croissant limite la concurrence avec les grandes entreprises européennes. De plus, les capitaux étrangers investis dans le secteur laitier ainsi que dans les autres secteurs d'activité menacent les entreprises polonaises.

La compétition est un défi incontournable pour les entreprises laitières dans le cadre de l'Union européenne. Il est certain que le but des restructurations du secteur laitier en Pologne, et en particulier des coopératives, est 
la création de structures compétitives bien organisées et bien gérées. Le processus de transformation de l'économie a détérioré le système de distribution. Certains canaux conventionnels de distribution (transformation, vente en gros, vente de détail) ont été abandonnés dans le secteur laitier. Un développement spontané du commerce privé a entrâné une grande rotation des marchandises sur le marché laitier. Peu de coopératives créent leur propre réseau de distribution et leurs canaux de vente. Elles se sont concentrées sur la stabilisation de leur position sur le marché local et l'entrée sur des marchés plus vastes. Le manque de canaux communs a causé une forte compétition entre les coopératives laitières. A l'avenir, il faudra accentuer les efforts pour créer une distribution commune, basée sur des circuits courts. Cela influencerait positivement les consommateurs, ainsi que les acteurs de la production. Un élément fondamental serait la création de postes coopératifs en gros et détail approvisionnant des clients grossistes et individuels. Grâce à ce modèle, des coopératives laitières pourront faire face à la compétition et renforceront leur position sur le marché [19]. De plus, de petites entreprises laitières retrouveront une activité positive. Trop souvent, elles n'ont pas coopéré pour prévenir la dégradation du système coopératif polonais, mais se sont au contraire fait concurrence pour s'écarter de petits marchés.

Le marketing peut être un facteur de développement. La stratégie marketing comprend la promotion des produits, leur emballage et la marque. Actuellement, en Pologne, peu de coopératives ont suffisamment de moyens pour développer ces activités. La faiblesse de la fonction marketing influe sur l'export. En effet, ne pouvant promouvoir des produits débanalisés, les entreprises produisent des marchandises non différenciées. Il est important que les coopératives puissent faire de la publicité dans les médias nationaux. Il est aussi nécessaire de créer un Centre d'information du marché et des prix [2]. L'Association des coopératives laitières de Basse Silésie a pris l'initiative de promouvoir des produits polonais et d'organiser une « marque polonaise " commune. Cette entreprise a pour objectif la création de liens verticaux entre les agriculteurs, les fournisseurs d'autres composants et d'entreprises de transformation et un réseau coopératif de distribution des produits sur le marché.

Le processus de concentration de la production et de la transformation du lait est inévitable. Il est impossible de maintenir le nombre actuel de coopératives laitières. En 2010, il devrait en rester 80-90 (la part des coopératives laitières dans l'achat de lait est estimée à plus de $80 \%$, et leur part dans la vente sur le marché, à plus de $70 \%$ ). Ainsi, moins d'un tiers des coopératives pourront se maintenir sur le marché. La concentration du secteur de la transformation permettra une spécialisation plus accentuée et des lignes de production plus longues. Ce secteur obtiendra plus facilement des moyens financiers pour sa modernisation et son développement, utilisera plus facilement les aides et connaitra une diminution des coûts. La réalisation du programme de restructuration du secteur laitier en Pologne, mené par l'Etat, nécessitera un financement à hauteur de 60 millions d'euros 
par an. Les fournisseurs, les entreprises de transformation, le budget national, les budgets de districts et d'autorités locales utiliseront des moyens trois fois plus élevés dans le même objectif. La consommation de lait et de produits laitiers devrait augmenter à l'horizon 2010. Les produits laitiers polonais pourront plus facilement être exportés et les coopératives polonaises seront plus fortes face à la concurrence des entreprises européennes.

\section{Propositions de solutions juridiques}

D'après les analyses juridiques effectuées sur le secteur coopératif polonais, la restructuration ne doit pas se limiter aux problématiques des coopératives, mais doit également s'étendre à toutes sortes d'unités de production. Une réforme des règles juridiques concernant les coopératives leur serait bénéfique dans la mesure où elle faciliterait l'intégration des unités de production à l'économie de marché et surtout à l'UE. Les changements proposés peuvent également conduire à une simplification des procédures de restructuration des coopératives.

En Pologne, la structure actuelle des coopératives (et en particulier des coopératives laitières) montre clairement le passage du modèle classique de coopérative, ne comptant que quelques membres, à celui de coopérative regroupant un grand nombre de fournisseurs. L'intégration et la concentration des entreprises ne doivent pas se faire sous forme de coopérative (coopérative de coopératives), mais par rassemblement de capitaux (sociétés à capitaux, convention de droit civil). Les coopératives peuvent aussi créer de grandes structures à caractère régional ou national par intégration des unités de production de la branche. Actuellement, il y a un réel désintérêt des membres de la coopérative quant à la capitalisation de leur coopérative du fait de la faible rentabilité de la production, mais également à cause du principe obligatoire admettant que chaque membre ait une seule voix indépendamment du nombre de parts sociales. Cette situation est notamment la conséquence de disparités très grandes dans les quantités apportées par les coopérateurs - les petits agriculteurs peuvent apporter quelques litres de lait, et les grosses exploitations, avoir quelques milliers de vaches laitières (anciennes grandes fermes d'Etat). Une solution peut consister à s'appuyer et à profiter des expériences de la France et des autres pays occidentaux pour accomplir une réforme du droit coopératif. Les différences essentielles existant entre certains aspects des coopératives françaises et polonaises sont présentées dans le tableau 9.

- En France, sur décision de l'assemblée générale, on peut introduire différentes options (objectif économique, objectif financier, gestion interne) dans les statuts de la coopérative pour rendre sa structure plus souple. Par exemple, l'option de gestion interne donne une possibilité de votes multiples. Une telle possibilité peut récompenser l'agriculteur qui a réalisé un grand chiffre d'affaires pour la coopérative. Cette manière de voter n'existe pas seulement dans le droit coopératif français, mais aussi dans plusieurs 
pays de l'UE. De telles clauses ont été revendiquées par les agriculteurs polonais, surtout par les gros fournisseurs, produisant des produits de bonne qualité (matière première). Il faut noter que, dans la pratique, cette option n'est quasiment jamais utilisée dans les coopératives de premier degré. Elle est surtout utilisée dans les unions de coopératives.

- Le montant du capital social, en France, est directement lié à l'importance des transactions réalisées avec la coopérative. Ainsi, les possibilités financières des coopératives augmentent et les membres se sentent plus responsables de l'avenir de leur coopérative. Le droit polonais permet une grande liberté dans ce domaine.

\section{Tableau 9}

Caractéristiques des entreprises coopératives en France et en Pologne

\begin{tabular}{|c|c|c|}
\hline Caractéristiques & France & Pologne \\
\hline Textes officiels & $\begin{array}{l}\text { - Loi } 47-1775 \\
\text { du } 10 \text { septembre } 1947 \\
\text { - Article } 1832 \text { et suivants } \\
\text { du Code civil } \\
\text { - Articles L } 521-1 \text { et suivants, } \\
\text { R 521-1 et suivants du Code rural: } \\
\text { - ordonnance du } \\
26 \text { septembre } 1967 \text {; loi } 72-516 \\
\text { du } 26 \text { juin } 1972 \text {; } \\
\text { - décrets } 59-286 \text { du } 4 \text { février } 1959 \\
\text { - modifié, } 78-704 \text { et } 78-705 \\
\text { du } 3 \text { juillet } 1978,84-407 \\
\text { du } 30 \text { mai } 1984\end{array}$ & $\begin{array}{l}\text { • Loi coopérative. } \\
\text { Texte uniforme } \\
\text { Journal législatifde: } \\
\text { 1982, n } 30 ; 1986, \mathrm{n}^{\circ} 39 ; \\
1987, \mathrm{n}^{\circ} 33 ; 1988, \mathrm{n}^{\circ} 41 ; \\
1989, \mathrm{n}^{\circ} 3 ; 1990, \mathrm{n}^{\circ} 6 ; \\
1991, \mathrm{n}^{\circ} 83 ; 1992, \mathrm{n}^{\circ} 21 ; \\
1994, \mathrm{n}^{\circ} 90\end{array}$ \\
\hline Objet & $\begin{array}{l}\text { - Utilisation en commun } \\
\text { par les agriculteurs des moyens } \\
\text { dans le but de faciliter } \\
\text { et de développer les activités } \\
\text { économiques de tous les membres }\end{array}$ & $\begin{array}{l}\text { - Mener des activités } \\
\text { économiques (production, } \\
\text { activités commerciales, services) } \\
\text { dans l'intérêt de ses membres }\end{array}$ \\
\hline $\begin{array}{l}\text { Associés } \\
\text { dits sociétaires }\end{array}$ & $\begin{array}{l}\text { - Personnes physiques ou morales } \\
\text { - Minimum sept coopérateurs; } \\
\text { pas de maximum } \\
\text { - Deux catégories: } \\
\text { - associés coopérateurs : } \\
\text { participent à la constitution } \\
\text { du capital; s'engagent à utiliser } \\
\text { les services de la coopérative; } \\
\text { ont droit aux ristournes } \\
\text { - associés non coopérateurs } \\
\text { (si option statutaire). }\end{array}$ & $\begin{array}{l}\text { - Personnes physiques ou morales } \\
\text { - Minimum cinq coopérateurs; } \\
\text { pas de maximum } \\
\text { - Minimum trois personnes morales } \\
\text { - Deux catégories: } \\
\text { - associés agriculteurs: } \\
\text { s'engagent à utiliser } \\
\text { les services de la coopérative; } \\
\text { ont droit aux ristournes } \\
\text { proportionnellement } \\
\text { à leur activité }\end{array}$ \\
\hline
\end{tabular}


Anciens associés coopérateurs, salariés, organismes financiers. Simples porteurs de part; n'ont pas d'activité économique; ne peuvent toucher de ristournes

- Votent en assemblée générale

- Sont représentés au conseil d'administration

\section{Formalités de constitution}

\section{Gestion}

- Rédaction des statuts (basée sur des statuts types homologués par le ministère de l'Agriculture) - Adoption des statuts par l'assemblée générale constitutive - Immatriculation au registre du commerce et des sociétés - Agrément par le ministère de l'Agriculture

- Publication au Journal officiel

- Administration:

- soit par un conseil d'administration composé d'associés coopérateurs et, en cas d'option statutaire, d'associés non coopérateurs dans une limite maximale;

- soit par un directoire et un conseil de surveillance - Contrôle des comptes par un commissaire aux comptes ou par un organisme de révision agréé

- Droit de vote équitable pour chaque membre. Possibilité de "pondération de voix " s'il existe une option statutaire - Principe d'exclusivité: il est possible de faire exception au règlement

(20\% des transactions)
- associés non agriculteurs (travailleurs journaliers de la coopérative): simples porteurs de part; n'ont pas d'activité économique avec la coopérative; ont droit aux ristournes tout comme les agriculteurs; pas de limite de voix à l'assemblée générale et au conseil de surveillance (traités de la même manière que les agriculteurs)

- Devient membre toute personne payant un minimum de capital social ou de droit d'enregistrement

- Rédaction des statuts (conformes aux lois coopératives) - Adoption des statuts par l'assemblée générale constitutive - Procédure conforme aux règlements juridiques des coopératives

- Enregistrement auprès de la justice - Immatriculation au registre du commerce et des sociétés - Publication de l'agrément au Journal officiel coopératif

- Administration: par un directoire et un conseil de surveillance

- Inspection par de vraies unions révisionnelles auxquelles ont adhéré les coopératives ou par l'inspecteur désigné par le conseil régional de la coopérative

- Droit de vote équitable pour chaque membre: un membre égale une voix. Pas de possibilité de "pondération de voix " comme dans le droit français - Responsabilité des associés limitée à la part de capital en tenant compte du montant apporté aux fonds sociaux

- Détermination et répartition des excédents annuels établis selon des obligations comptables

- Minimum $5 \%$ pour le fonds social, si ce fonds n'atteint pas 
- Responsabilité de l'associé:

- coopérateur, limitée à deux fois le montant des parts qu'il a souscrites ou aurait dû souscrire;

- non coopérateur, limitée à sa part de capital social

- Détermination et répartition des excédents annuels établis selon des obligations juridiques

- Dotations obligatoires à une réserve légale: $10 \%$ des résultats jusqu’à ce que la réserve soit égale au capital social

- Possibilité de réévaluation du bilan

\section{Particularités}

Régime fiscal
- L'action d'une coopérative est exercée dans une circonscription territoriale bien déterminée

- Nul ne peut faire partie de plusieurs coopératives agricoles pour le même service au titre de la même exploitation - Limitation au taux de rendement moyen des obligations de l'intérêt versé au capital souscrit par les associés coopérateurs et à deux points de plus pour celui souscrit par les associés non coopérateurs

- Dévolution, si dissolution, à des coopératives agricoles ou, avec l'assentiment du ministre de l'Agriculture, à des établissements d'intérêt général agricole - Tout sociétaire est tenu, pendant les cinq ans suivant son retrait, de toute dette sociale existant au moment de sa sortie

- Impôt sur les sociétés: exonération pour les opérations réalisées avec les sociétaires

- Taxe professionnelle:

- base réduite de moitié;

- exemption pour les coopératives employant trois salariés au maximum; pour les coopératives de vinification, Cuma, insémination animale la grandeur des participations obligatoires

- Possibilité de réévaluation du bilan

- L'action d'une coopérative est exercée dans une circonscription territoriale bien déterminée - En cas de liquidation, les associés ont le droit de revendiquer le capital social ou tout autre bien de la coopérative

- Impôt sur les sociétés semblable à celui des coopératives de droit commercial

- Pas d'exemption de taxes professionnelles 
- Dans les coopératives françaises, seuls les agriculteurs-membres peuvent réaliser des transactions avec la coopérative. Dans l'option « objectif économique ", le droit français permet de déroger au principe d'exclusivité. La coopérative peut réaliser des transactions avec des tierces personnes n'étant pas membres, mais seulement à hauteur de $20 \%$ des transactions. La loi polonaise ne fait aucune restriction, les coopératives mènent des activités commerciales qui souvent dépassent le revenu provenant de leurs activités de base prévues dans les statuts. Par exemple, la coopérative laitière fait du commerce de margarine, ce qui a beaucoup étonné les délégations françaises.

- Afin d'éliminer les actions néfastes et contraires aux intérêts des travailleurs de la coopérative et de ses membres, mieux vaut séparer précisément les fonctions de gestion de celles de propriétaire. La fonction de direction doit être confiée à des personnes choisies par les agriculteurs.

- Le montant du capital social détermine le domaine de responsabilité des membres. En France, il est déterminé jusqu’à la limite du double montant d'apport. Le montant du capital social est en général peu élevé. Ainsi, la perte totale ne peut être un facteur suffisant pour mobiliser les membres.

- En France, on observe un engagement des membres-agriculteurs dans la vie de la coopérative plus fort qu'en Pologne.

- La loi polonaise limite également la possibilité de dévolution du capital à « l'intérieur » de la coopérative. En effet, l'union des coopératives avec les unités de production privées est limitée. Il faut liquider la coopérative pour que son bien soit en totalité ou en partie transféré à une structure non coopérative. La loi française permet aux non-associés d'apporter du capital, mais ils ont très peu d'influence dans la gestion par rapport aux membres de la coopérative. Ils peuvent avoir jusqu'à $20 \%$ de voix à l'assemblée générale. Ils ne peuvent pas non plus profiter de ristournes.

Le résultat dans une coopérative n'a pas la même signification que dans une entreprise capitaliste. En effet, le conseil d'administration peut décider d'augmenter le prix payé aux agriculteurs et donc diminuer le résultat comptable. Il est donc logique que les coopératives ne soient pas redevables de l'impôt sur les sociétés. Seules les transactions menées avec les tiers sont imposées.

- Les régions d'implantation des coopératives sont spécifiées dans les statuts. Cela limite la concurrence interne entre les coopératives pour la matière première et permet de mener une politique commune pour les prix payés aux agriculteurs pour leurs produits.

- Le secteur coopératif français est équipé de différentes structures et organisations défendant et représentant les intérêts des coopérateurs. Il y existe un grand nombre d'unions nationales et régionales, ainsi que des groupes de coopératives adhérant directement à Coop de France. On remarque que les unités de production privées et les coopératives se rapprochent de plus en plus en formant des entités communes. Ces nouveaux groupes ont ainsi une plus grande force de négociation et une plus grande visibilité auprès 
des organisations représentatives du pouvoir (par exemple, Atla, créé en 1994 pour la branche laitière).

Lorganisation du secteur coopératif polonais est dispersée. Parmi les organisations coopératives, seules les coopératives laitières gardent leur position malgré une grande limitation des achats et de la production laitière. Pour les autres secteurs, les difficultés en termes d'organisation se maintiennent (font exception les banques coopératives). De plus, le type d'organisation de ces coopératives reste très éloigné des modèles occidentaux. L'organisation de notre secteur coopératif n'est pas cohérente. Bien que l'objet de chacune des coopératives figure distinctement dans les statuts, on remarque dans la réalité que leurs rôles et les tâches qu'elles accomplissent se recoupent. L'information ne circule pas assez efficacement entre ces organisations. Il n'y a pas de " cellule de communication » avec le milieu extérieur. Cette dispersion des efforts conduit aux conflits de compétences et à l'affaiblissement du secteur vis-à-vis des agents du gouvernement.

Pour que les coopératives puissent se maintenir dans ce contexte de forte transformation du système économique et s'opposer efficacement à la concurrence extérieure, elles doivent entamer quelques changements. Le projet de loi du 28 septembre 2004 en cours de discussion en Pologne permettra d'amorcer ces changements en modifiant certains aspects du statut actuel. 


\section{Bibliographie}

1. Agro Serwis nr 12/2001. Dodatek "Producent mleka w Unii Europejskiej" s. $18-19$.

2. Biuletyn informacyjny nr $4 / 2000$. Problemy integracji rolnictwa. Wyd. FAPA, s. 64.

3. Boss Rolnictwo "Branża mleczarska" 2001, s. 7.

4. Internet www.ppr.pl, "Sytuacja sektora mleczarskiego w Polsce", data 25-11-2002.

5. Internet www.ppr.pl, "Klasa extra", data 25-11-2002.

6. Internet www.ppr.pl, "Mleczny problem", data 25-11-2002.

7. Internet www.ppr.pl "Regulacja rynku mleka”, data 25-11-2002.

8. Internet www.zppm.com.pl, "O nas", data 3-12-2002.

9. Iwan B. "Przemiany spółdzielczości mleczarskiej”, s. 91-98.

10. Loi $n^{\circ} 91-5$ du 3 janvier 1991, dispositions relatives aux organismes coopératifs agricoles, annexe $\mathrm{n}^{\circ} 2$, Journal officiel de la République française.

11. Loi n ${ }^{\circ} 92-643$ du 13 juillet 1992 relative à la modernisation des entreprises coopératives, annexe $\mathrm{n}^{\circ} 2$, Journal officiel de la République française.
12. Mierzwa D., Organizacja sektora spółdzielczego we Fancji. Postęp Nauk Rolniczych, Nr 6, 1996.

13. Międzynarodowa Konferencja "Spółdzielczość wiejska w perspektywie integracji z UE”, s. 52-54.

14. Piróg W. : "Polska spółdzielnia w procesie przekształcenia gospodarczego. Wieś i Państwo, Nr 3, 1993.

15. RolnictwoaGospodarkaŻywnościowa w Polsce, Wyd. MRiGŻ, s. 10.

16. Rolniczy Rynek, nr 11/256, listopad 2001 Wyd. Regionalne Centrum Doradztwa, Rozwoju Rolnictwa i Obszarów Wiejskich we Wrocławiu. Artykuł pt. : "Organizacja Systemu Kwot Mlecznych w Unii Europejskiej”.

17. Rynek Mleka, kwiecień 2002. Wyd. IERiGŻ, Warszawa, s. 6-15.

18. Skawińska E. "Uwarunkowania rozwoju mleczarstwa polskiego w procesie integrowania Polski z UE” Tom I, Wyd. Toruńska Szkoła Zarządzania 1998.

19. Zalewski A., "Gospodarka mleczarska a rynek”. Wyd. IERiGZ̈, Warszawa 2000.

20. Mauget R., Koulytchizky S., "Le développement des groupes coopératifs depuis un demi-siècle, à la recherche d'un nouveau paradigme ", Recma, $n^{\circ} 287,2003$. 